\title{
Evaluation of the diagnostic value of the immunoblotting and ELISA tests using recombinant Em18 antigen in human alveolar echinococcosis from Xingjiang China
}

\author{
XIAO-JUAN BI ${ }^{1 *}$, YIN-MEI SHAO ${ }^{2 *}$, LIANG LI $^{1}$, YAN WANG ${ }^{1}$, CHUAN-SHAN ZHANG $^{1}$, GUO-DONG LÜ ${ }^{1}$, \\ TUERGANAILI AJI ${ }^{2}$, JUN LI ${ }^{1}$, WEN-BAO ZHANG ${ }^{1}$, HAO WEN $^{1}$ and REN-YONG LIN ${ }^{1}$ \\ ${ }^{1}$ State Key Laboratory of Pathogenesis, Prevention and Treatment of High Incidence Diseases in Central Asia, \\ Clinical Medicine Institute; ${ }^{2}$ Hepatobiliary \& Hydatid Department, Digestive and Vascular Surgery Centre, \\ The First Affiliated Hospital of Xinjiang Medical University, Urumqi, Xinjiang 830054, P.R. China
}

Received March 7, 2017; Accepted July 20, 2018

DOI: $10.3892 /$ etm.2018.6555

\begin{abstract}
Alveolar echinococcosis (AE) is a prevalent epidemic in the northern hemisphere, especially in central Europe and western China. Serum diagnosis is important for patients with AE, especially during the first screening. The present study purified the recombinant Em18-GST (rEm18-GST), and detected its diagnostic performance in human alveolar echinococcosis patients of Xinjiang, China with immunoblotting (IB) and enzyme-linked immunosorbent assay (ELISA). Serum samples were collected from 50 patients with AE, 222 patients with cystic echinococcosis (CE), 158 patients with other unrelated infections and 106 healthy individuals. The IB results showed that serum samples of 47 patients with AE and 12 patients with $\mathrm{CE}$ were rEm18-positive. However, only one sample from patients with cancer showed a cross-reaction with rEm18 in IB. The overall sensitivity was $94 \%$, and the total specificity was $96.58 \%$. For the rEm18 results using ELISA, the sera of 46 patients with AE were positive, and the overall sensitivity was $92 \%$. In conclusion, compared with imaging tools, including computed tomography, magnetic resonance imaging and positron emission tomography, rEm18 has considerable advantages for AE serodiagnosis.
\end{abstract}

Correspondence to: Professor Ren-Yong Lin, State Key Laboratory of Pathogenesis, Prevention and Treatment of High Incidence Diseases in Central Asia, Clinical Medicine Institute, The First Affiliated Hospital of Xinjiang Medical University, 137 Liyushan South Road, Urumqi, Xinjiang 830054, P.R. China

E-mail: renyong_lin@sina.com

*Contributed equally

Key words: alveolar echinococcosis, ELISA, immunoblotting, $\mathrm{rEm} 18$, serodiagnosis

\section{Introduction}

Alveolar echinococcosis (AE) is a lethal hepatic disease caused by the Echinococcus multilocularis (E. multilocularis) infection at the larval stage. Humans may get infected by swallowing the contaminated eggs. The disease is a prevalent epidemic in the northern hemisphere, especially in central Europe and western China $(1,2)$. The larval vesicles of the parasite propagate asexually in liver, the same as the tumor. Generally, the clinical symptoms could persist for a long time (up to 10 years), which usually causes liver dysfunction. At present, most AE patients are diagnosed with imaging methods, including radiology, magnetic resonance imaging, ultrasonography, and computed axial tomography (3-6). However, performing these methods in remote areas is relatively hard where the resources are limited. Thus, it is of great significance to diagnose AE patients in remote areas. Immunodiagnostic has been suggested as a possible method for screening a large population and as being suitable for diagnosis of AE $(7,8)$.

To identify and screen human AE, a 2-step procedure for diagnosis has been recommended by the World Health Organization (WHO) (9). First, Serologic screening is firstly conducted. The commonly used techniques included enzyme-linked immune sorbent assays (ELISAs), radio allegro sorbent tests (RAST), and indirect hemagglutination (IHA). Then, the results are further confirmed by the immunoelectrophoresis (IEP) or immunoblot (IB). Recently, Em18 has been isolated from the metacestode of the parasites (10), and was demonstrated to have high sensitivity and specificity for AE diagnosis through ELISA or IB $(8,11,12)$. However, these studies only included a relatively small panel of $\mathrm{AE}$ serum samples (approximately 20-30 AE patients). The value of recombinant Em18 (recEm18) in AE diagnosis with a large number of serum samples is not reported yet.

Here in this study, we have cloned and expressed the rEm18 in Escherichia coli (E. coli) as a GST fusion protein. A large number of serum samples were collected from patients in Xinjiang. Our aim was to clarify whether recEm18 is of high sensitivity and specificity in the serodiagnosis of AE, both by using IB and ELISA. 


\section{Materials and methods}

Patients and serum samples. Between March 2013 and December 2016, 536 serum samples (Table I) were enrolled at the First Affiliated Hospital of Xinjiang Medical University (Xinjiang, China). All protocols and usage of human sera in the study were approved by the Ethic Committee of the First Affiliated Hospital of Xinjiang Medical University. Fifty serum samples were from AE patients, who were diagnosed by surgery, imaging method, and serology analysis with a commercially available kit (Registration number 20153400177, Xinjiang Bestmind Bio Technology Development Co., Ltd, Urumqi, China) (7), and 222 serum samples were from CE patients who were confirmed by parasitological examinations after surgical removal. There were also serum samples collecting from patients with other unrelated parasitic diseases or nonparasitic diseases, including cysticercosis $(n=9)$, schistosomiasis $(n=7)$, paragonimiasis $(n=32)$, clonorchiasis $(n=20)$, cyst $(n=6)$, cancer $(n=8)$, or other disease $(n=76)$. The remaining samples were collected from healthy persons ( $\mathrm{n}=106)$, and 40 sera of them were used to calculate cut-off value.

Parasites and RNA extraction. Protoscolices (PSCs) were isolated from a Mongoliangerbils (Meriones unguiculatus) that had E. Multilocularis infection by intraperitoneal injection (i.p). After washing with PBS for 10 times, the PSCs were precipitated and aliquoted. The total RNA was then isolated from the PSCs with TRIzol reagent (Gibco; Thermo Fisher Scientific, Inc., Waltham, MA, USA).

Reverse transcription PCR and pGEM-T-Em-18 plasmid construction. The extracted RNAs were reverse transcribed into cDNA with a reverse transcription kit (SuperScript ${ }^{\mathrm{TM}}$ Preamplification System; Promega Corporation, Madison, WI, USA) per the manufacturer's instructions. To exclude the potential genomic DNA contamination, parallel reactions without reverse transcriptase were performed. The total volume for PCR was $50 \mu \mathrm{l}$, including $10 \mathrm{mM}$ Tris-HCl (pH 8.3), $50 \mathrm{mM} \mathrm{KCl}, 1.5 \mathrm{mM} \mathrm{MgCl}_{2}, 0.001 \%$ (wt/vol) gelatin, $0.1 \mu \mathrm{M}$ of each primer, $0.2 \mathrm{mM}$ of each deoxynucleoside triphosphate, $2 \mu \mathrm{cDNA}$, and 0.5 units of Taq DNA polymerase (Promega Corporation). The primer sequences were as follows: Up-stream primer, 5'-CCGGAATTCATGAAGGAG TCTGACTTAGCGGAT-3' and downstream primer, 5'-CCG CTCGAGTTTGAGGTTGGCCATCTTCGT-3'). The PCR conditions were $94^{\circ} \mathrm{C}$ for $5 \mathrm{~min}$ followed by 35 cycles of $94^{\circ} \mathrm{C}$ for $30 \mathrm{sec}, 55^{\circ} \mathrm{C}$ for $30 \mathrm{sec}$, and $72^{\circ} \mathrm{C}$ for $2 \mathrm{~min}$. After that, the product was cloned into a pGEM-T plasmid vector (Promega Corporation) and sequenced.

Sequence analysis. Sequencing data were analyzed using BLAST (http://www.ncbi.nlm.nih.gov/BLAST/). The predicted Em18 protein sequences of E. multilocularis and other parasites were aligned using CLUSTAL (http://www.ebi. ac.uk/clustalw/\#). The MEGA6.06 was used to construct the phylogenetic tree (13) and the neighbor-joining method (14) with 1,000 bootstrap replications was used.

Subcloning, expression, and purification of recombinant Em 18 (rEm18). The pET-41a (+)-Em18 plasmid was constructed by excising Em-18 sequence from thepGEM-T-Em-18 plasmid and ligating into the EcoRI and XhoI site of the pET-41a (+) expression vector (Novagen, Inc., Madison, WI, USA).The recombinant pET-41a (+)-Em18 plasmid was transformed into E. coli BL21 (DE3) cells (Novagen, Inc.). The expression of $\mathrm{rEm} 18$-GST fusion protein was induced with $1.0 \mathrm{mM}$ isopropyl - $\beta$-D-thiogalactopyranoside (IPTG) for $3 \mathrm{~h}$ at $37^{\circ} \mathrm{C}$. The protein was purified using GST binding resin under native conditions. At last, the purified protein was quantified by the Bradford assay. Bovine serum albumin was used as a standard sample.

Immunoblot analysis (IB). The rEm18-GST fusion protein was separated by $12 \%$ SDS-PAGE gels and then transferred onto a nitrocellulose membrane. After that, the membrane was cut into strips, with approximately $0.3 \mu \mathrm{g}$ of rEm18-GST on each strip. The strips were blocked with 5\% skim milk. Then, human serum samples (diluted 1:100) was added and incubated for $1 \mathrm{~h}$ at $37^{\circ} \mathrm{C}$. After rinsing with PBST for 3 times, the secondary antibodies of goat anti-human $\operatorname{IgG}$ conjugate with HRP (Sigma; Merck KGaA, Darmstadt, Germany) were added and incubated for $2 \mathrm{~h}$ at room temperature. Finally, the strips were incubated with diaminobenzidine (DAB) for $15 \mathrm{~min}$ at room temperature for color development.

ELISA. ELISA were performed using plates coated with rEm18. Briefly, microtitration plates (Nalge Nunc International, Roskilde, Danmark) were coated with $100 \mu$ l of antigen solution $(1 \mu \mathrm{g} / \mathrm{ml})$ per well in carbonate/bicarbonate buffer ( $\mathrm{pH} 9.6)$ at $4{ }^{\circ} \mathrm{C}$ overnight. After washing PBST for three times, the plate was incubated with $5 \%$ skim milk for $1 \mathrm{~h}$ at $37^{\circ} \mathrm{C}$. After washing again, $100 \mu \mathrm{l}$ of serum sample (1:100 dilution) was added into each well and incubated at $37^{\circ} \mathrm{C}$ for $1 \mathrm{~h}$. Then, $100 \mu \mathrm{l}$ of secondary antibody (peroxidase-conjugated rabbit anti-human immunoglobulin G (IgG) (Sigma; Merck KGaA) was added, and the mixture was incubated for $1 \mathrm{~h}$ at $37^{\circ} \mathrm{C}$. Finally, $100 \mu \mathrm{l}$ of substrate was added to each well and incubated for $15 \mathrm{~min}$ at $37^{\circ} \mathrm{C}$. The optical density at $450 \mathrm{~nm}\left(\mathrm{OD}_{450}\right)$ was measured with the ELISA plate reader (Bio-Rad Laboratories, Inc., Hercules, CA, USA). Serum samples were recorded as positive if the OD values were greater than the three times of the OD450 values for 40 healthy normal controls.

Statistical analysis. The values of sensitivity, specificity, and positive and negative predictive values were calculated. Fisher's exact test was used for comparison among groups. A $\mathrm{P}<0.05$ was considered to be statistically significant.

\section{Results}

Molecular cloning and characterization of Em18. The Em18 gene sequence (GenBank accession no. AY513691.1), which comprised a 486-bp coding region, was amplified with the specific primers by PCR from cDNA of E. multilocularis PSCs. Em18 protein contains 161 amino acid and has a molecular mass of $18.3 \mathrm{kDa}$. Based on the sequence homology analysis, the recEm18 was similar to recEm18 from Sichuan (GenBank accession no. AAS00619.1) with only three different amino acids. Meanwhile, comparative analysis between Em18 and Eg18 from the Xinjiang isolate (GenBank accession no. AY513265.1) revealed an identical nucleotide 
Table I. Characteristics of patients and healthy individuals.

\begin{tabular}{|c|c|c|c|c|}
\hline Characteristics & $\mathrm{AE}(\mathrm{n}=50)$ & CE (n=222) & Other diseases $(n=158)$ & Healthy individuals $(n=106)$ \\
\hline $\begin{array}{l}\text { Age at diagnosis (years), } \\
\text { mean } \pm \text { standard deviation }\end{array}$ & $44.6 \pm 15.7$ & $49.6 \pm 11.6$ & $39.0 \pm 16.0$ & $46.2 \pm 10.7$ \\
\hline \multicolumn{5}{|l|}{ Sex, n (\%) } \\
\hline Male & $19(37.5)$ & $75(33.7)$ & $80(50.6)$ & $44(41.6)$ \\
\hline Female & $31(62.5)$ & $147(66.3)$ & $78(49.4)$ & $62(58.3)$ \\
\hline \multicolumn{5}{|l|}{ Source of drinking } \\
\hline \multicolumn{5}{|l|}{ Water, n (\%) } \\
\hline Well & $25(50.0)$ & $115(51.8)$ & - & - \\
\hline Spring & $20(40.0)$ & $74(33.3)$ & - & - \\
\hline River & $3(6.0)$ & $12(5.4)$ & - & - \\
\hline Tap & $2(4.0)$ & $21(9.5)$ & - & - \\
\hline \multicolumn{5}{|l|}{ Dog in the family } \\
\hline Yes & $23(46.0)$ & $77(34.7)$ & - & - \\
\hline No & $27(54.0)$ & $145(65.3)$ & - & - \\
\hline \multicolumn{5}{|c|}{ Previous history of hydatidosis } \\
\hline Yes & $14(28.0)$ & $55(24.8)$ & - & - \\
\hline No & $36(72.0)$ & $167(75.2)$ & - & - \\
\hline
\end{tabular}

AE, alveolar echinococcosis; CE, cystic echinococcosis.

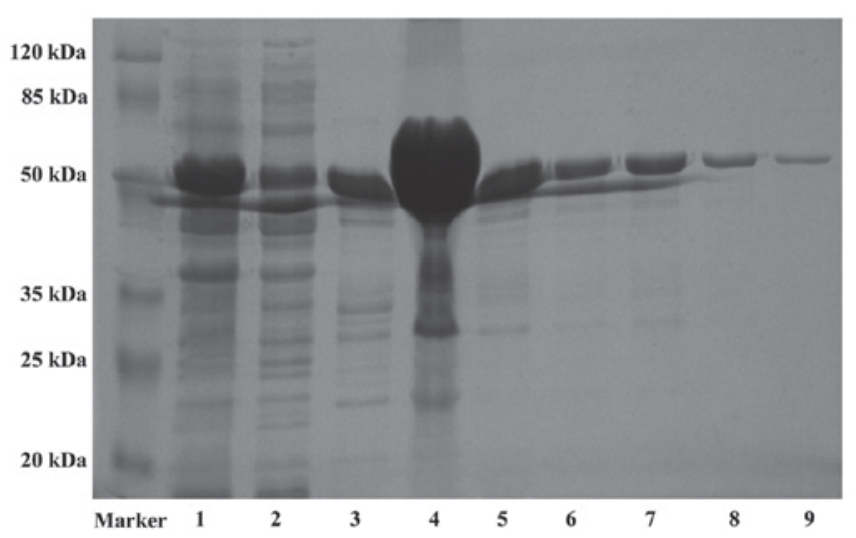

Figure 1. SDS-PAGE analysis of recombinant Em18. SDS-PAGE was performed to detect the expression of the recombinant Em18 glutathione $S$-transferase (GST) fusion protein (rEm18-GST). Lanes 1-9: Purified protein of rEm18 -GST from first to ninth elution.

sequence. The pET-41a (+)-Em18 plasmid was constructed and the expression of rEm18-GST was induced with IPTG. After that, the fusion protein was purified through GST affinity beads, which was approximately $51 \mathrm{kDa}$ (Fig. 1). These results indicate that Em18 from Sichuan and Xinjiang have little difference in the amino acid sequence.

Phylogenetic comparison of Em18 and Eg18. The neighbor joining method was used to construct a phylogenetic tree. As shown in Fig. 2, Em18 was closely related to Eg18, Eg10, EmII/3 as well as E. granulosus tegument protein, and Moesin/ezrin/radixin protein. However, the homology of this recombinant antigen with human and other parasitic moesin family proteins was the lowest. Thus, the phylogenetic data suggested that AE patients can be easily distinguished from patients contaminated infected with other parasites by rEm18.

Diagnostic performance of rEm18-GST by IB and ELISA. ELISA (Fig. 3) and IB (Fig. 4) methods were used to evaluate the diagnostic value of the purified rEm18-GST fusion protein. The serum samples were collected from AE patients. As shown in Table II, rEm18-GST showed positive reactions with $94 \%(47 / 50)$ of serum samples from AE patients, as revealed by IB testing, and the ratio was $92 \%(46 / 50)$ by ELISA. For patients who had unrelated diseases, a total of $3.42 \%$ of the serum samples $(n=380)$ had positive reaction with $\mathrm{rEm} 18-\mathrm{GST}$, among which, 5.41\% (12/222) of serum samples from CE patients cross-reacted with the recEm18-GST. One sample from lung cancer patient who have not the particular history, also cross-reacted with rEm18-GST. However, no positive results were observed with serum from patients with neurocysticercosis $(n=9)$, patients with diseases $(n=149)$, or healthy individuals $(n=106)$. The statistical significance were not observed between IB and ELISA groups $(\mathrm{P}>0.05)$.

\section{Discussion}

Alveolar echinococcosis (AE) causes high chronic morbidity and mortality in Central Asia, European countries, North/Latin America and northwest China (15). One of the clinical features of tissue-invasive larval cestodiases is slow progression with minimal symptoms and signs, unless infected parasites provoke acute symptoms (16). Early diagnosis of AE can significantly improve the both quality of disease diagnosis and treatment. The multiple serological studies have been 


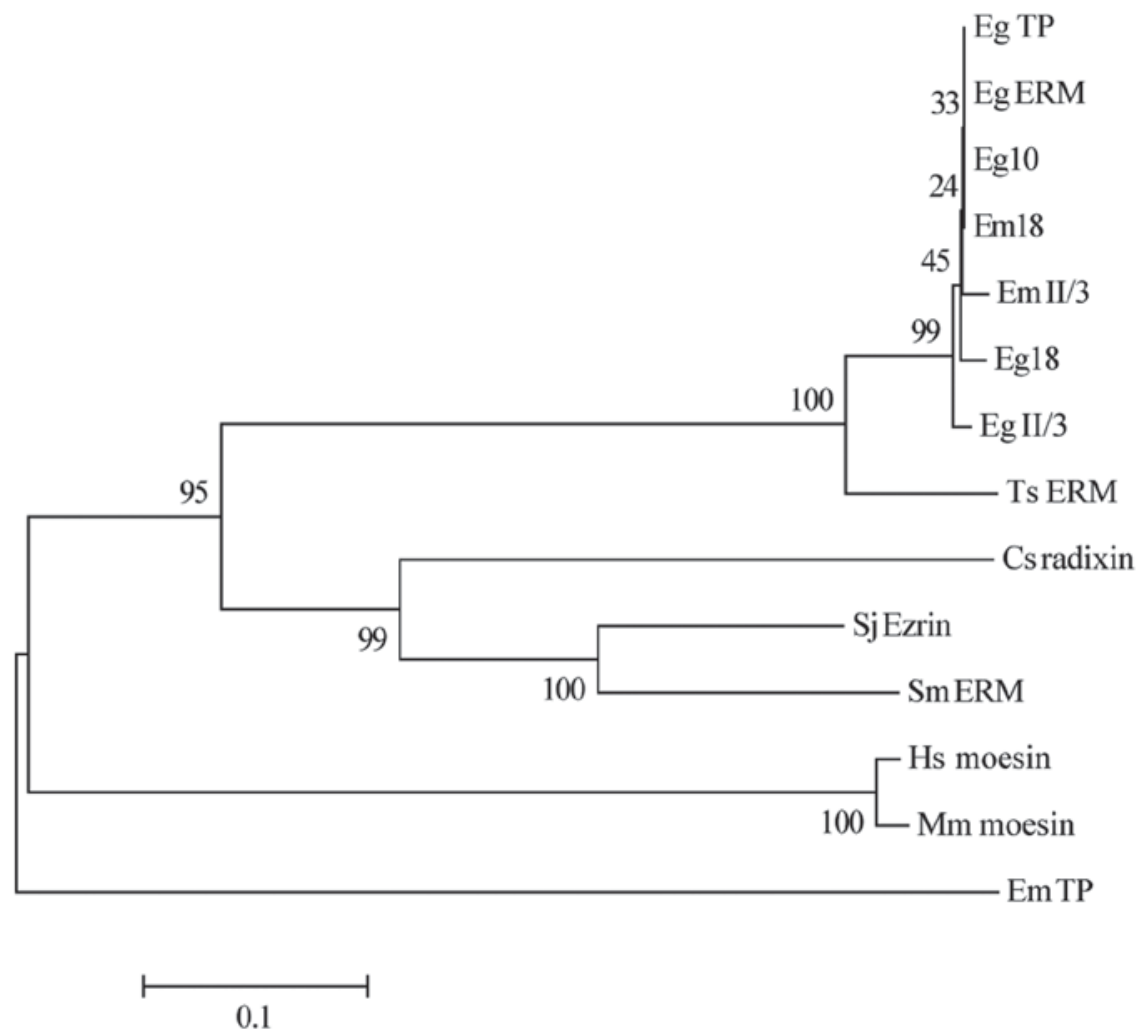

Figure 2. Phylogenetic tree for consensus of Em18 sequences and relative reference sequences by Neighbor-joining method. The phylogenetic tree was constructed using the neighbor-joining method with MEGA6.06 based on the amino acid alignment (Clustal W). The bootstrap percentage values of 1,000 replicates are indicated at each branch node. The GenBank accession numbers of the analyzed sequences are as follows: EmTP, E. multilocularis tegument protein, AAA29063.1; EmII/3, E. multilocularis antigen II/3, AAA50580.1; EgII/3, E. granulosus, antigen II/3, AAA50580.1; Em18, E. multilocularis, AAR99825.1; Eg18, E. granulosus, AAS00620.1; Ts ERM, Taenia solium ezrin-radixin-moesin-like protein, BAI49989.1; Eg10, E. granulosus, CAA82625.1; Sj Ezrin, Schistosoma japonicum Ezrin, CAX72657.1; Sm ERM, Schistosoma mansoni merlin/moesin/ezrin/radixin protein, CCD82162.1; EgTP, E. granulosus tegument protein, CDS19541.1; Eg ERM, E. granulosus Moesin/ezrin/radixin protein, EUB64035.1; Cs radixin, Clonorchis sinensis radixin, GAA42464.2; Hs moesin, Homo sapiens moesin, NP_002435.1; and Mm moesin, Mus musculus moesin, NP_034963.2.

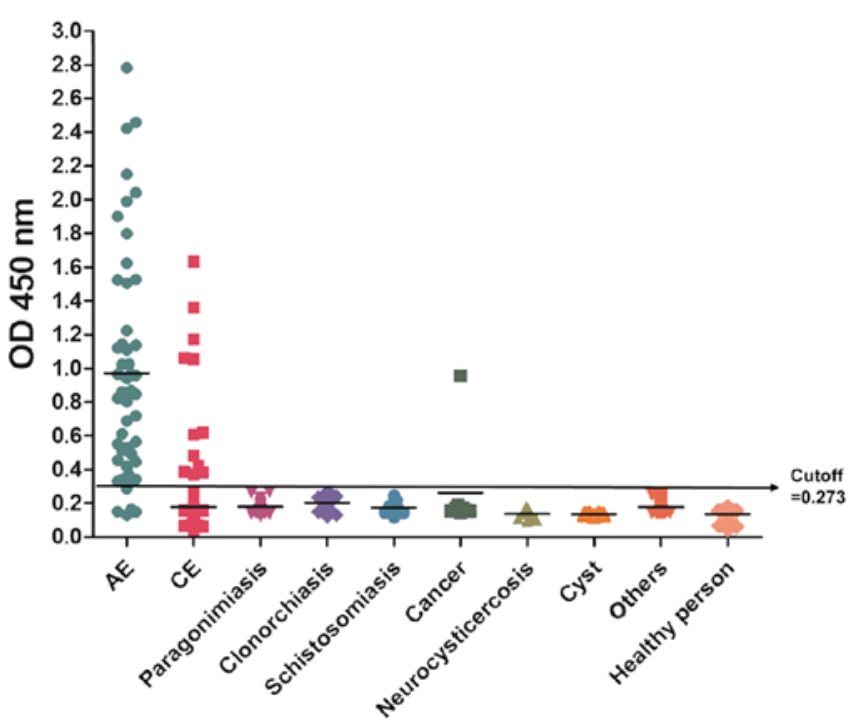

Figure 3. Diagnostic performance of rEm18-GST by ELISA. The cutoff value was defined as the value that was three times of the OD value of the negative control. AE $(n=50), C E(n=222)$, paragonimiasis $(n=32)$, Clonorchiasis $(n=20)$, Schistosomiasis $(n=7)$, Cancer $(n=8)$, Neurocysticercosis $(n=9)$, Cyst $(n=6)$, Otherdisease $(n=76)$, Healthy person $(n=106)$.

carried out that serology can be used to detect early (asymptomatic) cases in populations living in endemic areas $(6,17,18)$.
Immunodiagnostic of $\mathrm{AE}$ is doubtlessly one of the most valuable diagnostic methods in the early detection of $\mathrm{AE}$ infection (19). In most cases, these methods are cheap, relatively easy to use, and are necessary for large-scale screening in high-risk groups (20). Here, the fusion protein rEm18-GST was constructed and purified. The role of rEm18-GST in the early diagnosis of AE was investigated by IB and ELISA.

Em2 is isolated from the metacestode of E. multilocularis (21). As a species-specific natural antigen, Em2 has been widely used for serodiagnostic studies (22) and has shown encouraging results in the immunodiagnostic of human AE (23). However, the sensitivity of Em2 in ELISA, with range of 77 to $92 \%$, is dependent on the patient's geographical origin. The works on other recombinant E. multilocular molecules have also been undertaken for the diagnostic purposes, and the results are encouraging. The Em2 plus ELISA, which is a combination of Em2 with a II/3-10recombinant protein, has increased the sensitivity to $97 \%$ (21). However, the Em2 plus assay has also shown a cross-reaction to CE (in 25.8\% of cases), which is higher than that for individual Em2 (5.6\%) or II/3-10 (6.5\%), respectively. To explain why these similar proteins have distinct immunogenicity in $\mathrm{AE}$ and $\mathrm{CE}$ patients, Sako et al (10) have speculated that E. multilocularis may contact invasively and intimately with host tissues. For this reason, in CE patients, B-cell responses to Em18 might be low. Further studies are needed to analyze the relationship of the 
Table II. Diagnostic performance of rEm18-GST for human AE by IB and ELISA.

Positive serum samples, no. $(\%)$

Serum samples

No.

Em18-IB

Em18-ELISA

AE

$\begin{array}{lrrr}\text { Surgery }^{\mathrm{a}} & 31 & 29(93.55) & 28(90.32) \\ \text { Image analysis }^{\mathrm{b}} \text { plus serology } & 19 & 18(94.74) & 18(94.74) \\ \text { CE } & & & 3(2.83) \\ \quad \text { Surgery }^{\mathrm{a}} & 106 & 9(7.76) & 9(7.76) \\ \text { Image analysis }^{\mathrm{b}} \text { plus serology } & 116 & 0(0) & 0(0) \\ \text { Neurocysticercosis } & 9 & 0(0) & 0(0) \\ \text { Paragonimiasis } & 32 & 0(0) & 0(0) \\ \text { Clonorchiasis } & 20 & 0(0) & 0(0) \\ \text { Schistosomiasis } & 7 & 1(12.5) & 1(12.5) \\ \text { Cancer } & 8 & 0(0) & 0(0) \\ \text { Cyst } & 6 & 0(0) & 0(0) \\ \text { Other } & 76 & 0(0) & 0(0) \\ \text { Healthy person } & 106 & & \end{array}$

Percentages were calculated as follows: Sensitivity $=[\mathrm{tp} /(\mathrm{tp}+\mathrm{fn})] \times 100=47 / 50=94.0 \%$ by IB and $92 \%$ by ELISA, respectively; specificity $=[\mathrm{tn} /(\mathrm{tn}+\mathrm{fp})] \mathrm{x} 100=(380-13) / 380=96.58 \%$ by WB and ELISA, respectively; tp represents true positives, fn represents false negatives, fp represents false positives, and tn represents true negatives. Serum samples were recorded as positive if the $\mathrm{OD}_{450}$ values were higher than three times the $\mathrm{OD}_{450}$ values of human serum samples pooled from 40 healthy Chinese adults. The cutoff value is 0.273 . a Serum samples of patients confirmed by surgery. ${ }^{b}$ Serum samples of patients confirmed by image analysis plus serology with a commercially available kit. IB, immunoblotting; ELISA, enzyme-linked immunosorbent assay; AE, alveolar echinococcosis; CE, cystic echinococcosis; Em, Echinococcus multilocularis; No., number; rEm18-GST, recombinant Em18 GST fusion protein; OD, optical density.

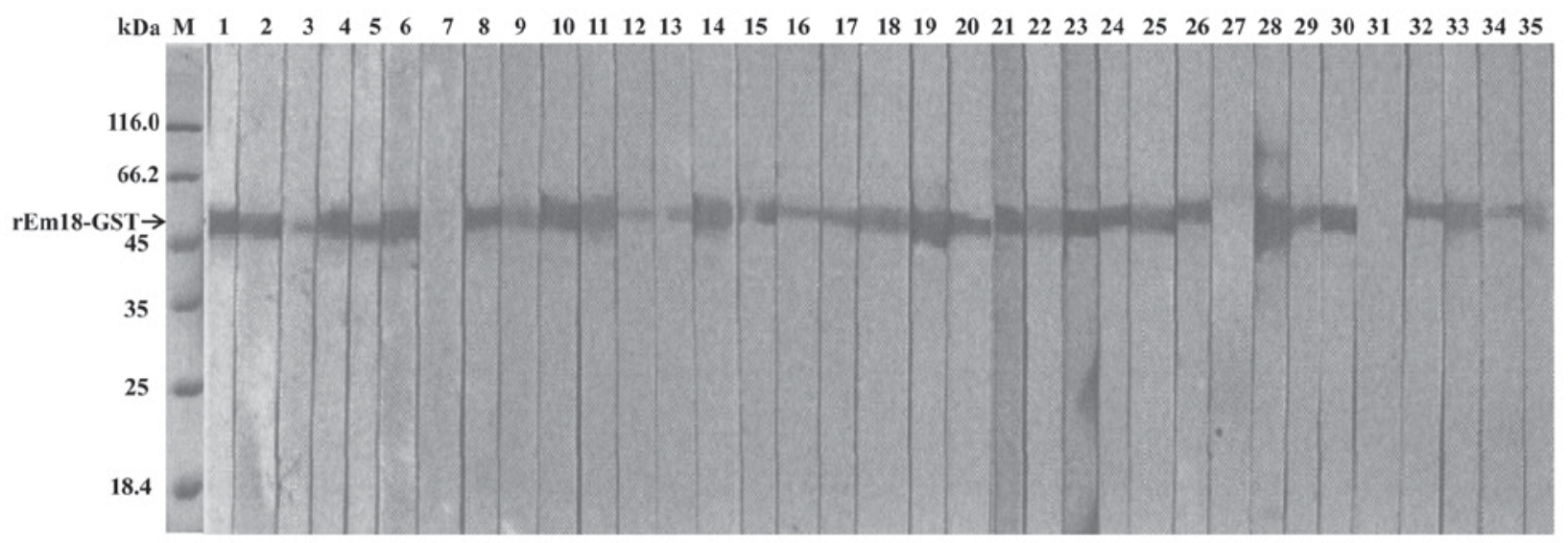

Figure 4. Immunoblotting analysis of rEm18-GST with 35 representative serum samples from AE patients. The black arrow indicates the rEm18-GST band.

B-cell epitopes and Em18. The serodiagnostic performance of rEm13 protein (sensitivity and specificity) has also been analyzed $(24,25)$, however, only a small numbers of serum samples were tested. Our results here suggest that the diagnostic performance of rEm18-GST by both IB and ELISA may be better than other reported reagents, particularly when a large number of serum samples were tested.

To sum up, our results suggest that rEm18 diagnosis is both easy to use and cheap. This method may be used to confirm the clinical findings of AE and a follow-up to surgery or pharmacological therapy (26). And, this method has the minimum requirement for equipment and time, especially during the first screening.

\section{Acknowledgements}

Not applicable.

\section{Funding}

This work was supported by the Xinjiang Tianshan Innovation Team Program (grant no. 201705120), The 
XinjiangYouth Science and Technology Innovation Talent Training Project-Xinjiang Outstanding Youth Natural Science Foundation Project (grant no. QN2016JQ0327) and the National Natural Science Foundation of China (grant nos. 81560330, 81371838, 81760368 and 81660341).

\section{Availability of data and materials}

The datasets used and/or analyzed during the current study are available from the corresponding author on reasonable request.

\section{Authors' contributions}

RYL designed and directed the experiment. XJB, LL, YW and CSZ performed the experiments. XJB, GDL and HW performed the statistical analysis. YMS and TA contributed in collecting clinical samples. JL and WBZ provided Mongolian gerbils infected with Em18 and performed the isolation of protoscolices. XJB and YW wrote the manuscript. JL, WBZ, HW and RYL reviewed and edited the manuscript. All authors read and approved the final manuscript.

\section{Ethics approval and consent to participate}

Ethical approval for the study was granted from the Ethic Committee of the First Affiliated Hospital of Xinjiang Medical University and all patients gave written informed consent.

\section{Patient consent for publication}

All patients provided written informed consent for the publication of their data.

\section{Competing interests}

The authors declare that they have no competing interests.

\section{References}

1. Torgerson PR, Keller K, Magnotta M and Ragland N: The global burden of alveolar echinococcosis. PLoS Negl Trop Dis 4: e722, 2010.

2. Zhang W, Zhang Z, Wu W, Shi B, Li J, Zhou X, Wen H and McManus DP: Epidemiology and control of echinococcosis in central Asia, with particular reference to the People's Republic of China. Acta Trop 141: 235-243, 2015.

3. McManus DP, Gray DJ, Zhang W and Yang Y: Diagnosis, treatment, and management of echinococcosis. BMJ 344: e3866, 2012.

4. Bresson-Hadni S, Delabrousse E, Blagosklonov O, Bartholomot B, Koch S, Miguet JP, Mantion GA and Vuitton DA: Imaging aspects and non-surgical interventional treatment in human alveolar echinococcosis. Parasitol Int 55 (Suppl): S267-S272, 2006.

5. Azizi A, Blagosklonov O, Lounis A, Berthet L, Vuitton DA, Bresson-Hadni S and Delabrousse E: Alveolar echinococcosis: Correlation between hepatic MRI findings and FDG-PET/CT metabolic activity. Abdom Imaging 40: 56-63, 2015.

6. Romig T, Kratzer W, Kimmig P, Frosch M, Gaus W, Flegel WA, Gottstein B, Lucius R, Beckh K and Kern P: An epidemiologic survey of human alveolar echinococcosis in southwestern Germany. Römerstein Study Group. Am J Trop Med Hyg 61: 566-573, 1999

7. Feng X, Wen H, Zhang Z, Chen X, Ma X, Zhang J, Qi X, Bradshaw H, Vuitton D and Craig PS: Dot immunogold filtration assay (DIGFA) with multiple native antigens for rapid serodiagnosis of human cystic and alveolar echinococcosis. Acta Trop 113: 114-120, 2010.
8. Knapp J, Sako Y, Grenouillet F, Bresson-Hadni S, Richou C, Gbaguidi-Haore H, Ito A and Millon L: Comparison of the serological tests ICT and ELISA for the diagnosis of alveolar echinococcosis in France. Parasite 21: 34, 2014.

9. Poretti D, Felleisen E, Grimm F, Pfister M, Teuscher F, Zuercher C, Reichen J and Gottstein B: Differential immunodiagnosis between cystic hydatid disease and other cross-reactive pathologies. Am J Trop Med Hyg 60: 193-198, 1999.

10. Sako Y, Nakao M, Nakaya K, Yamasaki H, Gottstein B, Lightowers MW, Schantz PM and Ito A: Alveolar echinococcosis: Characterization of diagnostic antigen Em18 and serological evaluation of recombinant Em18. J Clin Microbiol 40: 2760-2765, 2002.

11. Tappe D, Sako Y, Itoh S, Frosch M, Grüner B, Kern P and Ito A: Immunoglobulin $\mathrm{G}$ subclass responses to recombinant Em18 in the follow-up of patients with alveolar echinococcosis in different clinical stages. Clin Vaccine Immunol 17: 944-948, 2010.

12. Ito A, Nakao M and Sako Y: Echinococcosis: Serological detection of patients and molecular identification of parasites. Future Microbiol 2: 439-449, 2007.

13. Li X, Zheng T, Zheng X, Han N, Chen X and Zhang D: Molecular characterization of two fatty Acyl-CoA reductase genes from phenacoccus solenopsis (Hemiptera: Pseudococcidae). J Insect Sci 16: pii: 49, 2016.

14. Saitou N and Nei M: The neighbor-joining method: A new method for reconstructing phylogenetic trees. Mol Biol Evol 4: 406-425, 1987.

15. Ahn CS, Bae YA, Kim SH, Kim JG, Yu JR, Yang HJ, Eom KS, Wang H, Kang I, Yang Y and Kong Y: Spatiotemporal expression patterns and antibody reactivity of Taeniidae endophilin B1. J Clin Microbiol 54: 2553-2562, 2016.

16. Cecconi A, Maroto L, Vilacosta I, Luaces M, Ortega L, Escribano N, Vivas D, Ferreirós J, Montes L, Vilchez JP, et al: Acute pericarditis secondary to hydatid cyst rupture: Diagnosis by multimodality imaging. Circulation 128: 2073-2074, 2013

17. Gottstein B, Saucy F, Deplazes P, Reichen J, Demierre G, Busato A, Zuercher C and Pugin P: Is high prevalence of Echinococcus multilocularis in wild and domestic animals associated with disease incidence in humans? Emerg Infect Dis 7: 408-412, 2001.

18. Bresson-Hadni S, Laplante JJ, Lenys D, Rohmer P, Gottstein B, Jacquier P, Mercet P, Meyer JP, Miguet JP and Vuitton DA: Seroepidemiologic screening of Echinococcus multilocularis infection in a European area endemic for alveolar echinococcosis. Am J Trop Med Hyg 51: 837-846, 1994.

19. McManus DP, Zhang W, Li J and Bartley PB: Echinococcosis. Lancet 362: 1295-1304, 2003.

20. Zhang W, Li J and McManus DP: Concepts in immunology and diagnosis of hydatid disease. Clin Microbiol Rev 16: 18-36, 2003.

21. Pektaş B, Altintaş N, Akpolat N and Gottstein B: Evaluation of the diagnostic value of the ELISA tests developed by using EgHF, Em2 and EmII/3-10 antigens in the serological diagnosis of alveolar echinococcosis. Mikrobiyol Bul 48: 461-468, 2014 (In Turkish).

22. Deplazes P and Gottstein B: A monoclonal antibody against Echinococcus multilocularis Em2 antigen. Parasitology 103: 41-49, 1991.

23. Gottstein B, Deplazes P and Aubert M: Echinococcus multilocularis: immunological study on the 'Em2-positive' laminated layer during in vitro and in vivo post-oncospheral and larval development. Parasitol Res 78: 291-297, 1992.

24. Frosch PM, Geier C, Kaup FJ, Müller A and Frosch M: Molecular cloning of an echinococcal microtrichal antigen immunoreactive in Echinococcus multilocularis disease. Mol Biochem Parasitol 58: 301-310, 1993.

25. Qi X, Liu Y, Wei W, Huang X and Zuo Y: Effects of the C-terminal of endostatin on the tumorigenic potential of $\mathrm{H} 22$ cells. Biomed Rep 1: 761-765, 2013.

26. Sako Y, Tappe D, Fukuda K, Kobayashi Y, Itoh S, Frosch M, Grüner B, Kern P and Ito A: Immunochromatographic test with recombinant Em18 antigen for the follow-up study of alveolar echinococcosis. Clin Vaccine Immunol 18: 1302-1305, 2011. 